\title{
Urethral Adenocarcinoma
}

National Cancer Institute

\section{Source}

National Cancer Institute. Urethral Adenocarcinoma. NCI Thesaurus. Code C6167.

An adenocarcinoma that arises from the male or female urethra. 